\title{
The Minimax Sphere Eversion
}

\author{
G. Francis ${ }^{1}$, J. M. Sullivan ${ }^{2}$, R. B. Kusner ${ }^{3}$, K. A. Brakke ${ }^{4}$, \\ C. Hartman $^{1}$, G. Chappell ${ }^{1}$ \\ 1 Mathematics Dept., University of Illinois, Urbana-Champaign, IL \\ 2 Mathematics Dept., University of Minnesota, Minneapolis, MN \\ 3 Mathematics Dept., University of Massachusetts, Amherst, MA \\ 4 Mathematics Dept., Susquehanna University, Selinsgrove, PA
}

February 1996; published in Visualization and Mathematics, eds. Hege \& Polthier, Springer, 1997, pp. 3-20 plus color plates.

\begin{abstract}
We consider an eversion of a sphere driven by a gradient flow for elastic bending energy. We start with a halfway model which is an unstable Willmore sphere with 4 -fold orientation-reversing rotational symmetry. The regular homotopy is automatically generated by flowing down the gradient of the energy from the halfway model to a round sphere, using the Surface Evolver. This flow is not yet fully understood; however, our numerical simulations give evidence that the resulting eversion is isotopic to one of Morin's classical sphere eversions. These simulations were presented as real-time interactive animations in the CAVE automatic virtual environment at Supercomputing'95, as part of an experiment in distributed, parallel computing and broad-band, asynchronous networking.
\end{abstract}

\section{A History of Sphere Eversions}

To evert a sphere is to turn it inside out by means of a continuous deformation, a regular homotopy, which allows the surface to pass through itself, but forbids more serious singularities where the curvature becomes locally infinite. There have been many eversions since Smale [30] proved the possibility of this phenomenon forty years ago. The extraordinary difficulty in visualizing an explicit eversion has made it an effective challenge for a succession of both mathematical and graphical innovations. 
Our sphere eversion differs from all previous ones in that it proceeds automatically through an optimization procedure. We use the Surface Evolver, a computer program which is designed to solve variational problems, like finding the shape of soap films. We needed only to specify the starting point and a general strategy of energy minimization to the program. The resulting minimax eversion is optimal in that it requires the least bending at any stage.

As in all good experiments, we did not know a priori that the evolver would be successful in producing a sphere eversion. To our great pleasure, it not only succeeded, but also produced an eversion which turned out to be among those designed by Bernard Morin three decades earlier [28]. That an optimal geometry for the eversion would match one envisioned by a pure topologist is an eloquent confirmation of mathematics.

The sphere eversion story has been told many times from a variety of viewpoints. Chapter 6 of [9] discusses most of the work through 1986 primarily from the viewpoint of how to draw pictures of a process. For an update as well as a good review of the topological fundamentals, see Silvio Levy's supplement [22] to the video Outside In [23].

Here we focus on computer animations, beginning with Nelson Max's epic $16 \mathrm{~mm}$ film [25]. It was generated as a frame-by-frame animation from a database of coordinates in $\mathbb{R}^{3}$ which had been entered manually [26]. The coordinates were taken from wire-mesh models made by C. Pugh, which depict, in eleven stages, what is to be perceived as a continuous surface moving through itself from a highly convoluted halfway model to a round sphere. This halfway model is an example of a Morin surface, an immersed sphere which has a 4-fold rotational symmetry that switches its orientation. (Thus if the two sides of this sphere were painted different colors, the quarter-turn would bring the surface back to its original position, but with the colors exchanged.) Morin recognized that a homotopy from a round sphere to such a surface automatically gives a sphere eversion; the second half of the eversion can be obtained from the first by reversing the temporal order, rotating the models $90^{\circ}$ and switching colors. The resulting eversion has one kind of temporal symmetry; a fundamentally different kind figured in the first explicit sphere eversion (by Arnold Shapiro [12]) and the first illustrated publication of an eversion (by Tony Phillips [29]). These use the double cover of a Boy surface as a halfway model, and thus the symmetry exchanges only time and color.

In 1977, Morin created an analytic parametrization [27] of his eversions. While it was conceptually correct, its implementation on a computer was visually unrecognizable. The first real-time, interactive computer animation was produced by John Hughes on a Stardent [19]. He fit spherical harmonics to polyhedral models of key stages in Morin's eversion, obtaining a global parametrization by trigonometric polynomials. Morin and Denner developed a minimally polyhedral eversion [2], and Apéry [1] calculated harmoniously scaled algebraic formulas for its smooth companion. Both of these eversions were implemented as real-time interactive animations [11]. 
A truly new idea for an eversion, Bill Thurston's corrugations [22], was expertly realized and stunningly rendered in the 1994 Geometry Center videotape, Outside In [23]. Unlike the earlier eversions, this method furnishes a general recipe for creating regular homotopies between surfaces whenever this is known to be theoretically possible. However, for all of these eversions, their designers had to know a priori what they should look like, and then search for formulas capturing this behavior. This limits the usefulness of their software tools in cases when one does not already know the whole story. We set about to build tools that were exploratory as well as explanatory. Our minimax eversion is generated automatically, with even the topological structure being chosen by the optimization procedure.

\section{The Minimax Sphere Eversion}

Kids climbing fences, along with engineers building mountain roads and scientists rocketing to the moon, know that the easiest way to get from one side to the other (and back again) is to follow the path which goes over the lowest spot. This is so obvious to kids that they don't have a name for (or at least they don't tell their parents about) this spot on a fence, but of course it is usually called a pass or saddle on roads through the mountains.

It is precisely such a lowest-energy saddle that we encounter halfway through turning a sphere inside-out via the minimax sphere eversion. Indeed, the minimax sphere eversion might be viewed as the "easiest" path of immersed spheres leading from a round sphere with the right side out to one inside-out. The energy which climbing kids and road engineers care about is the height they need to go above the surrounding territory. For mathematicians interested in everting spheres, another energy is needed: the elastic bending energy, which assigns to any immersed surface the integral of the square of the mean curvature. This energy is often called $W$, after Tom Willmore, who rekindled interest in $W$ among mathematicians in the 1960s [31].

In 1983, Allen Hatcher [15] proved the Smale Conjecture, which in one formulation asserts that the diffeomorphism group of $\mathbb{R}^{3}$ is homotopy equivalent to the orthogonal group $O(3)$. An equivalent formulation says that the space of embedded spheres in $\mathbb{R}^{3}$ is contractible. Interested in finding an analytic proof of this, Kusner started looking for functions with nice gradient flows on the configuration space of embedded (or immersed) surfaces.

That $W$ might be a good candidate follows from Robert Bryant's [7] result: the only embedded $W$-critical sphere is round. The strategy for proving the Smale Conjecture analytically would be to flow an embedded sphere down the $W$-gradient until the sphere stopped changing, and thus, was round. Unfortunately, this flow does not preserve embeddedness. ${ }^{1}$ Bryant

\footnotetext{
1 A gradient flow for $W$ would correspond to a fourth-order parabolic equation, which unlike a second-order equation, does not enjoy a maximum principle.
} 
had also found immersed $W$-critical spheres with self-intersections. Understanding these variationally led Kusner quite naturally to the idea of the minimax sphere eversion, because of some further important results already known about $W$.

Willmore's main result [31] was that $W$ is uniquely minimized by round spheres, with the value $4 \pi$; any other surface $M$ has energy $W(M)$ greater than $4 \pi$. When the surface is immersed, there is a more general bound (due to Li and Yau [24], sharpened in [20]):

If there is a point of $\mathbb{R}^{3}$ through which $k$ "sheets" of a surface $M$ pass, then $W(M)$ is at least $4 k \pi$; equality can occur here only if there is a complete minimal surface $\tilde{M}$ in $R^{3}$ with $k$ planar ends ("sheets at $\infty$ "), and a Möbius transformation which carries $\tilde{M}$ to $M$ (and $\infty$ to the $k$-fold point on $M$ ).

Taking $k=1$ and $\tilde{M}$ to be a flat plane, this implies Willmore's original result. The proof makes use of the fact that the quantity $W+4 k \pi$ (where $k$ is the multiplicity of the surface at $\infty$ ) is invariant under Möbius transformations of $\mathbb{R}^{3} \cup \infty$.

Bryant's classification [7] of $W$-critical spheres ${ }^{2}$ showed that the lowest saddle point for $W$ is at $16 \pi$, realized by a certain family of immersed spheres with one quadruple point. By the above, each such surface arises as the Möbius transformation of some minimal surface in $\mathbb{R}^{3}$ with four planar ends.

In the meantime, Tom Banchoff and Nelson Max had shown [3] (see also [18]) that every sphere eversion must pass through an immersed sphere with at least one quadruple point. Thus, by the Li-Yau inequality above, every sphere eversion must pass over the $W=16 \pi$ level. And if some $W$ critical sphere at this $16 \pi$ level were a saddle point (rather than a local minimum), then we could simply flow to either side of the saddle (in the most negative Hessian direction) by a $W$-gradient flow, and the flow would have to proceed down to the $W$-minimizing round sphere on either side. Note that if the saddle surface halfway model has an orientation-reversing symmetry, then these two round spheres must have the opposite orientation. So, by climbing up the (positive) $W$-gradient flow, over the saddle and back down the other side, one would get an optimal sphere eversion: the minimax sphere eversion.

In 1977 Francis circulated an illustrated manuscript, see [9, Ch. 6], about sphere eversions equivariant under all the cyclic rotation groups, the tobacco pouch eversions. This inspired Kusner to find an infinite family of $W$-critical spheres (with even-order cyclic symmetry) and real projective planes (with odd-order symmetry). These are Möbius images of complete minimal surfaces with planar ends for which there is explicit, symmetric Weierstrass data [20]. They include a $W$-minimizing Boy surface with 3 -fold symmetry, as well as the Morin surface of 4-fold symmetry that we use as our halfway model $h_{0}$.

\footnotetext{
2 Bryant's proof can be simplified and unified using the spinor representation and an abstract skew-form [21].
} 
The image $M$ of this immersion $h_{0}$ is a Möbius transformation of a minimal surface $\tilde{M}$ whose Weierstrass representation can be integrated explicitly. The surface $\tilde{M}$ is given as an immersion $\tilde{h}$ of the Riemann sphere $\mathbb{S}^{2}$ with four punctures into $\mathbb{R}^{3}$ :

$$
\tilde{h}(w)=\mathfrak{R e}\left(\frac{\left(i\left(w^{3}-w\right),\left(w^{3}+w\right), \frac{i}{2}\left(w^{4}+1\right)\right)}{w^{4}+2 \sqrt{3} w^{2}-1}\right) .
$$

From this formula, we can check that if $\tilde{h}(w)=(x, y, z)$, then $\tilde{h}(\bar{w})=$ $(-x, y,-z)$ and $\tilde{h}(i / w)=(-y,-x,-z)$. Thus the 4 -fold symmetry of the Riemann sphere given by the action of $w \mapsto i / \bar{w}$ appears as an orientationreversing 4 -fold rotational symmetry $\rho_{4}$ of $\tilde{M}$ around the $z$-axis. The poles $w=0$ and $w=\infty$ both map to the origin, and no other points map to the $z$-axis. The minimal surface $\tilde{M}$ has four planar ends, corresponding to the four punctures - the values of $w$ where the denominator vanishes.

A Möbius transformation takes $\tilde{M}$ to a compact surface $M=h_{0}\left(\mathbb{S}^{2}\right)$, which will be a critical point for the elastic energy with $W=16 \pi$. We want our halfway model $h_{0}$ to still have the 4 -fold rotational symmetry $\rho_{4}$. Therefore we use a Möbius transformation which is an inversion around a point on the $z$-axis: $(x, y, z) \mapsto\left(x / r^{2}, y / r^{2}, s+(z-s) / r^{2}\right)$, where $r^{2}=$ $x^{2}+y^{2}+(z-s)^{2}$. There is no particular reason to choose a specific value of $s \neq 0$. We chose $s=0.35$ in our experiments for aesthetic reasons. The four sheets at infinity in $M$ have become a quadruple point of $h_{0}$ at $(0,0, s)$; the two tangent sheets of the surface originally at the origin are now at $(0,0, s-1 / s)$, the isthmus point of $h_{0}$.

The minimax eversion is now given by a heteroclinic orbit ${ }^{3}$ for the $W$ gradient flow, from the saddle point $h_{0}$ down to the global minimum, a round sphere. Since this gradient flow takes infinitely long, we rescale time so that $t=0$ is the halfway model and we reach the round sphere at $t=1$. The Euclidean gradient for $W$ is given by a nonlinear fourth-order operator. Hence our flow will certainly exist for a short time, but there is no guarantee of long-time existence. Our experiments suggest, however, that the flow indeed exists from our halfway model to the round sphere. The complete eversion is a homotopy $h_{t}: \mathbb{S}^{2} \rightarrow \mathbb{R}^{3}$, for $t \in[-1,1]$ with the temporal symmetry $h_{-t}(w)=\rho_{4}\left(h_{t}(-i / \bar{w})\right)$, and $h_{-1}$ and $h_{1}$ are round spheres that differ by the antipodal map.

Our minimax eversion is optimal in the sense that it requires the least bending energy at any intermediate stage. While we cannot yet construct this eversion theoretically, we can compute it numerically. In the next section we show how to compute an approximation of the homotopy $h_{t}$ described above, replacing the halfway model by a nearby polyhedral surface $\mathcal{H}_{0}$. The continuous motion in time is replaced by a sequence of small steps, $\mathcal{H}_{n}$, leading to a polyhedral approximation of a round sphere.

\footnotetext{
3 A flowline leading from one critical point to another (in doubly infinite time) is called a heteroclinic orbit.
} 


\section{Using the Surface Evolver to evert a sphere}

In 1986, when Kusner computed some pictures ${ }^{4}$ of the possible halfway models, there was no software available to compute the entire gradient flow. About that time, Brakke started work on the Surface Evolver [5], an interactive computer program in the public domain ${ }^{5}$ for the study of surfaces shaped by various energies and constraints. The evolver uses a finite element method, with a surface being represented as a set of triangles in space. There are no restrictions on orientation or topology, hence complicated surfaces such as foams may also be handled. Originally available energies included surface tension and gravity. Constraints can include fixed volumes and boundary conditions. The usual action of the evolver is to minimize total energy by gradient descent. ${ }^{6}$

At the 1991 Five Colleges Regional Geometry Institute, Kusner directed the research program on computation in geometric analysis, where Brakke and his evolver were star attractions. We urged Brakke to add features to let surfaces minimize energies like $W$, instead of just surface area. Indeed, that summer Brakke worked out, and programmed into the evolver, formulas for several discretizations of $W$ and their gradient flows.

Lucas Hsu, Kusner and Sullivan performed extensive experiments [17] minimizing $W$ for surfaces of higher genus; some of the resulting surfaces were animated in [16]. In 1992 at MSRI, Kusner and Sullivan presented this work on Willmore surfaces, and Francis presented joint work with Apéry on the Morin-Denner cuboctahedral sphere eversion [11,2]. We decided then to work on animating the minimax sphere eversion with the evolver.

To obtain the discrete halfway model $\mathcal{H}_{0}$, we start by evolving a sphere. We can start with an octahedron, fix the enclosed volume, and repeatedly refine the facets (replacing each triangle by four) and iterate the conjugategradient descent for surface area. Since the sphere of course is the least-area way to enclose that volume, the evolver quickly approaches a sphere. We wrote a short program to read in one evolver datafile and move all the vertices by the inverted Weierstrass map $h_{0}$. Applying this to a sphere of about 1500 facets gives a polyhedral halfway model. Next we switch to minimizing $W$ (or really, a discretization $\mathcal{W}$ ), and no constraints are needed.

Because the map $h_{0}$ is conformal, the triangulation of this halfway model is still nearly equilateral, though globally uneven in size. We start by using the evolver's query language to selectively refine long edges to get a more even triangulation; the equiangulation command, which selectively swaps diagonals, is also helpful throughout in preserving a nice triangulation. If

\footnotetext{
${ }^{4}$ With Michael Callahan and David Hoffman at GANG, the Center for Geometry, Analysis, Numerics and Graphics at the University of Massachusetts, Amherst.

5 Available at URL http://www.geom.umn.edu/locate/evolver/.

6 Actually, we use the conjugate gradient method because it is better at following narrow sloping valleys downhill in high dimensions.
} 
we now iterate (with gradient-descent steps for $\mathcal{W}$ ), we get an even better approximation $\mathcal{H}_{0}$ to the halfway model.

We are near an unstable critical point for the energy. Repeated iteration would drive us closer for quite some time, but eventually the unstable mode would presumably dominate, and we would fall off the saddle and down towards a round sphere. However, this would take much too long. The evolver can instead compute the Hessian of the energy $\mathcal{W}$. We could use this, in Newton's method, to converge to the unstable critical point, but in practice there are so many eigenvalues close to zero (from the Möbius degrees of freedom, or tangential motions of vertices) that this is difficult.

The Hessian calculation confirms our suspicion that $\mathcal{H}_{0}$ has Morse index one, meaning there is only one unstable direction; if 4-fold symmetry is enforced, we are at a local minimum for $\mathcal{W}$. Since our initial sphere had octahedral symmetry, our polyhedron $\mathcal{H}_{0}$ has 4 -fold symmetry. This is important, for we want to generate the negative-time half of the eversion using this symmetry, with no glitch in the triangulation. But it means we would have to wait until some numerical noise crept in to see any motion away from the saddle point. Thus, to get the evolution started, we instead use another new feature of the evolver: it can compute the most negative eigenvalue of the Hessian, and move in the direction of the corresponding eigenvector. Our initial step is made in this direction. Theoretically, after one such saddle step, we could now use conjugate-gradient descent. But in practice, since we are still in a regime of small gradient, it seems best to repeat the following sequence: every fifty iterations of descent are followed by another saddle command and retriangulation. (Note that saddle computes the Hessian, or quadratic term in the Taylor polynomial for $\mathcal{W}$; then it finds the line of the lowest eigenvector and chooses the downhill direction along this line.)

The retriangulation just mentioned involves several commands, all under automatic control. We refine any edges with high dihedral angle with the notch command, and refine any long edges. We equiangulate a couple of times, and delete (or coalesce) small edges and facets (and even slightly larger ones in flat regions of the surface). The evolver script to do this is

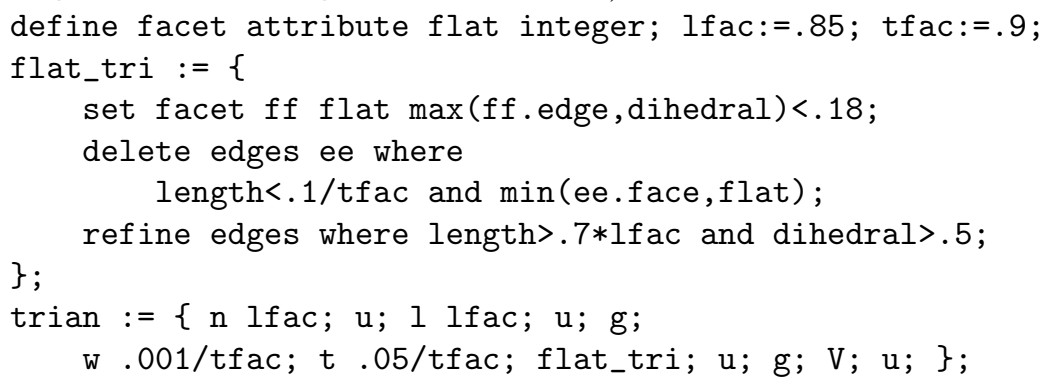

where the constants $l f a c$ and $t f a c$ are changed at a later stage of the evolution, where fewer facets are necessary.

Once we are further from the saddle point, we can proceed without the saddle commands, except at one later stage of the homotopy. At the 
gastrula stage (described below), the surface looks like a sphere connected to a smaller sphere by a catenoidal neck. Since spheres and catenoids are both critical points for $W$, most regions of this surface see no reason to move, and would take much too long to progress through this stage with conjugate gradient alone; saddle (again used every fifty steps) helps the neck grow.

Usually the evolver is run with interactive three-dimensional graphics shown in geomview. ${ }^{7}$ The picture is normally updated every time the surface changes, but this approach is unsuitable for our present purposes. On an ordinary machine, the eversion will take hours, and is best done in batch mode. On a multiprocessor PowerChallenge from Silicon Graphics, we can compute the eversion in about 10 minutes, but sending graphics of each step would slow this down too much. Instead, we turn off the automatic display and instead show the graphics only once every fifty iterations (just before the retriangulation and possible saddle). With this choice, we get about 126 topes, the polyhedral stages of the discrete homotopy $\mathcal{H}_{n}$, which are saved as 3 -D object display lists. For $n=126$ we are very near a round sphere. The number of facets stays between 1000 and 2000 throughout; we find this is enough to be confident we are approximating a smooth surface, while the computation still proceeds at a tolerable speed.

The evolver saves topes in the common file format OFF, and these can be viewed in geomview and other viewers. We have, however, written a special purpose viewer, illiVert. ${ }^{8}$ This application of our evolving illiView ${ }^{9}$ prototype of a real-time interactive CAVE animation (RTICA), can display actual or saved evolver output either on an Iris console, or in one of the immersive virtual environments described below. It has special features for coloring and shrinking facets, which make it easier to view the homotopy, especially in binocular stereo.

One theoretical difficulty with the $W$-gradient flow is that the usual versions are not Möbius-invariant. This means that if we started with a halfway model differing by some Möbius transformation, the resulting flow would be truly different; it might even have different topological stages. This difficulty also appears in our evolver experiments: because the discretization $\mathcal{W}$ is Möbius-invariant, the evolver might compose the gradient motion at any stage with some Möbius transformation. Thus, especially towards the end, where large portions of the surface are spherical, and have little reason to

\footnotetext{
7 Available at URL http://www.geom.umn.edu/locate/geomview/.

8 See URL http://new.math.uiuc.edu/laterna/ for a copy of illiVert that shows the minimax eversion on any Iris.

9 The illiView collection of real-time interactive computer animations is produced and maintained by Francis and his students in courses taught in the Renaissance Experimental Laboratory, a donation from SGI to NCSA. Its software design philosophy, its niche within mathematical visualization, and its pedagogical implications were discussed in $[14,10]$. The principal feature of an illiView prototype, which facilitates rapid adaptation to new hardware innovations, is its simplicity and conceptual optimization.
} 
move, we sometimes see jumps or drifts in our animation, due to added Möbius transformations.

We plan next to improve the computation of the eversion by making use of new symmetry features of the evolver [6]. These will allow us to enforce 2 -fold rotational symmetry throughout the evolution, making computations with just half of the surface. Furthermore, by enforcing 4-fold symmetry, we can get an even better halfway model $\mathcal{H}_{0}$. We could start with any surface of this symmetry, like the halfway model ${ }^{10}$ from the cuboctahedral eversion [2] mentioned above; by minimizing $\mathcal{W}$, we would approach $h_{0}$ (up to the choice of the parameter $s$ in the Möbius transformation).

Enforcing symmetry throughout the eversion will also tend to reduce the jumps we see from added Möbius transformations; the surface is only free to drift along the transformations that preserve the $z$-axis. With the extra speed and quality of this symmetric evolution, we plan to create a highquality narrated video, using a significantly finer triangulation and storing many more topes.

\section{A Topological Description of the Eversion}

As we have seen, the evolver produces a sequence of polyhedral sphere immersions $\mathcal{H}_{n}$, the topes of the minimax sphere eversion. This sequence starts with the triangulated approximation $\mathcal{H}_{0}$ to the smooth halfway model, $h_{0}: \mathbb{S}^{2} \rightarrow \mathbb{R}^{3}$ obtained by inversion from the Weierstrass representation of an immersed minimal surface. We imagine that the $\mathcal{H}_{n}$ approximate a regular homotopy, $h_{t}$. However, we do not know a formula for any such $h_{t}$, and changing the script used by the evolver might approximate an isotopic deformation of $h_{t}$. Thus we shall let $h_{t}$ denote the hypothetical regular homotopy we think we are seeing when watching the animation of the $\mathcal{H}_{n}$; here $n=0,1, \ldots, N$ is assumed to be injected monotonically into $0 \leq t \leq 1$.

We next describe $\mathcal{H}_{0}$ and its set of self-intersection curves, also called the double locus, in terms of what can be discerned from Color Plates 8 and 9 , pp. 20, 19 in the Appendix, and from the line drawings here. However, we also intend this description to be a guide to a real-time interactive session with illiVert.

The top row of Color Plate 8 shows three views of the halfway model. In the left image, we look straight down the symmetry axis at the origin in $\mathbb{R}^{3}$, which we call the pole point on the minimax halfway model, since $h_{0}$ maps both poles, $z=0, \infty$ of $\mathbb{S}^{2}$ there. Directly below this, also on the $z$-axis, is the quadruple point, which is of considerably greater interest; the middle image shows the halfway model from the bottom, with the quadruple point in view. The right image shows a cut-away view from the side, demonstrating how convoluted the halfway model really is.

\footnotetext{
10 This polyhedral model of Morin's surface is minimal in the sense that it has the fewest possible vertices, 12 , and faces, 14 .
} 


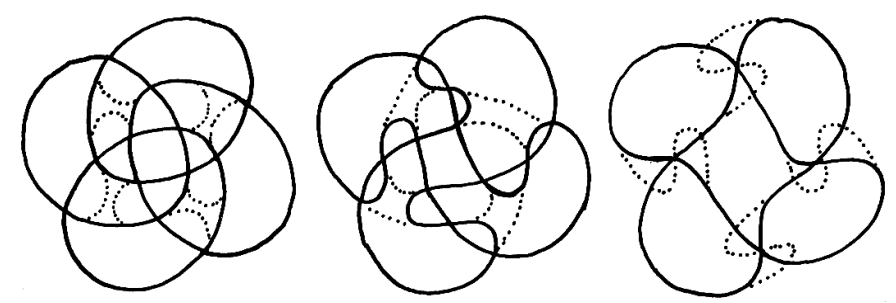

Fig. 1 Generic cross-sections of the halfway model below (left) and above (middle) the four saddles, below (middle) and above (right) the four pitchfork bifurcations.

In Color Plate 9 we show three stereo pairs ${ }^{11}$ of cut-away views of the halfway model from the top. In the top pair, we have moved the horizontal clipping plane just past the pole point and can see the quadruple point. The missing piece of the surface consists of two identical elongated bowls, their rims artfully bent (like integral signs) to fit, and oppositely colored. Note that even the slightest perturbation will destroy the pole point. However any generic motion of one bowl through the other passes through a singularity in the double locus which is isotopic to the present one. The canonical model for this type of generic catastrophe in a regular homotopy, denoted by $D_{1}$, is the pair of surfaces $z=x y-t, z=0$. Since this looks like the inundation of a saddle sinking below the water table it is known as an isthmus event $[25]$.

The quadruple point, on the other end, is formed by four bowls in 4-fold symmetry, as seen in the bottom stereo pair of Color Plate 9. Since there are four minima and two maxima for this height function on our sphere, elementary Morse Theory predicts four saddle points, which are visible in the middle pair, near $11,2,5$ and 8 o'clock. We have traced the three generic levels of $\mathcal{H}_{0}$ in a line drawing, Fig. 1, to emphasize the salient features.

To trace the curves of self-intersection, or double locus, follow the 12 curves emanating from the quadruple point. ${ }^{12}$ Before we reach the pole point, eight of them have disappeared pairwise, somewhere between the top and the middle stereo pair. This occurs at four additional $D_{1}$ isthmus events of the sphere eversion. We describe these in terms of the customary visualization of the classical pitchfork bifurcation shown in Fig. 2.

The wavy surface in the middle image is the cubic $y+x^{3}+z x=0$; the planar one is $y=0$. The horizontal slices as $z$ varies show an S-shaped curve crossing a line three times at first, and later just once. Even the slightest perturbation, like $y=t$, breaks the pitchfork in one of two ways, as in the left or right pictures. Hence a regular homotopy passing through a pitchfork suffers a generic $D_{1}$-catastrophe. What cannot be seen, but

\footnotetext{
11 These can be viewed by crossing the eyes so that the left image is seen with the right eye and vice versa.

12 There are two, one above the other, in each of the four principal directions, as well as one in each diagonal direction.
} 

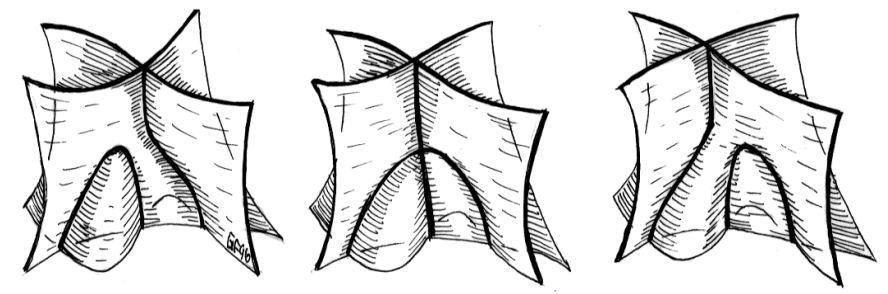

Fig. 2 A planar surface patch crosses a cubic patch along a double locus that looks like a 3-pronged pitchfork. Even the slightest perturbation breaks the pitchfork into two curves, one bent into a hairpin, the other more or less continuing vertically.

perhaps imagined, is the way the four pitchforks break as $\mathcal{H}_{t}$ evolves off the minimax saddle. ${ }^{13}$

In the evolver and in illiVert, we intentionally preserved 2-fold spatial symmetry about the axis, and temporal symmetry, in the sense that $\mathcal{H}_{-t}=\rho_{4} \mathcal{H}_{t}$. So one bowl is bound to expand while the other bowl contracts. The $D_{1}$ pitchforks break oppositely and reaveal two orifices (as in the second row of Color Plate 8) on opposite sides of the object. With illiVert you can fly into those orifices and look around the interior of the model.

Perhaps it is appropriate here to explain why we did not say "the inside of the model" at the end of the previous paragraph. A simplicial complex embedded in 3-space is perceived as a model of something. If the facets are small enough, their dihedrals are close enough to a straight angle, and they are rendered with smooth shading, ${ }^{14}$ then we are tricked into seeing an object composed of continuously bending surface patches. But we cannot be expected to see immediately that these patches are logically connected in such a way as to admit a parametrization (even if hypothetical) by a sphere.

To orient ourselves let us begin at the end of the eversion, $t=-1$, when the model looks undeniably like a sphere, painted yellow-orange on one side and purple-cyan on the other. ${ }^{15}$ The first part of the eversion is an isotopy that results in a shape that is best described as two concentric spheres connected by a hyperbolic neck. With Apéry, we find the embry-

\footnotetext{
13 Our experimental polyhedral approximation to the eversion shows these pitchforks happening in pairs, two near tope $\mathcal{H}_{-4}$ and two near tope $\mathcal{H}_{4}$. However, as pointed out by Nick Schmitt, our smooth halfway model $h_{0}$ has four double tangencies, so in the true minimax eversion, these four $D_{1}$ events happen simultaneously at $t=0$. The intersection behavior of minimal surfaces indicates that the two sheets at each double tangency must disagree to second order. This makes the events look more like the quadratic isthmus than the cubic pitchfork.

${ }^{14}$ We did not use the standard Gouraud smooth shading precisely to emphasize the facets, and to aid binocular stereo vision.

15 The precise color within each range is determined by the angle between the facet and the axis, making self-intersections easier to see.
} 
ological metaphor apt, whereby a spherical blastula invaginates to form the gastrula, third row right of Color Plate 8. The 'hole' or stoma ultimately becomes the mouth (neck) of the organism. At this stage, the embedded, non-convex sphere has elastic bending energy approximately twice that of the round sphere. The neck contributes little or no energy, as it is close to a catenoidal minimal surface. The inside of the blastula has become the thin space between the inner and outer sheets of the gastrula. Also, there is now an interior and an exterior in the colloquial sense, connected by the single orifice.

The embryological analogy eventually fails, at some time $t=-t_{7}$, when, instead of forming an orifice at the other end of the intestinal tract, the inner sphere penetrates the outer one forming a circle of double-points and a lens shaped chamber, as in row 3 of Color Plate 8. This type of catastrophe in a regular surface homotopy is called the birth of a double curve and is denoted by $D_{0}$. It is here, at the caudal end of the model, that the polar isthmus $D_{1}$ catastrophe will eventually occur, at halftime, $t_{0}=0$.

A second $D_{0}$ occurs far removed from the first, spatially and temporally for the minimax eversion. ${ }^{16}$ The mouth of the gastrula, bottom right of Color Plate 8 , sheds its nearly circular symmetry for a 2 -fold symmetry, in row 3 of Color Plate 8 . As diagonally opposite saddles move tailward the two umbilical patches between the saddles turn towards the axis. The $D_{0}$ occurs as they penetrate each other at $t=-t_{6}$ and form a second circle of double-points. From the outside, the model now looks like a cashew. The erstwhile 'inside' of the sphere has become a toroidal space, and a second lens-shaped chamber is formed at the head of the model. Now there are two orifices from the exterior to the interior of the model, but note that both the exterior and the interior are on the same side of the immersed surface.

There is one further catastrophe between the cashew and the halfway model, occurring at $t=-t_{5}=-t_{4}$ and shown in the second row of Color Plate 8 . The symmetric birth of triple point pairs, denoted by $T^{+}$, is easy enough to describe locally. Consider a model, shown in Fig. 3, consisting of two convex patches intersecting along a vertical double-curve, and crossing the horizontal floor at two further U-shaped double-curves making two triple points with the vertical curve. As the floor rises, the triple points die together in a catastrophe denoted by $T^{-}$, leaving two separating doublecurves. The reverse of this homotopy creates the triple point pair at a $T^{+}$.

We can now describe the evolution of the double locus, isolating it from the model, in purely topological terms. Two initially disjoint (and unlinked) circles, come to touch in two places forming two triple point pairs. In a spatially quite complicated manner, the four triple points converge to the quadruple point. The local model of the $Q$ catastrophe is that of the four planes of a tetrahedron moving orthogonally through its barycenter, Fig. 4.

\footnotetext{
${ }^{16}$ Clearly, the two $D_{0}$ catastrophes could have occurred at the same time, $t_{6}=$ $t_{7}$, or even in reverse order. Without symmetry constraints, they could occur in many other places on the gastrula. This accounts for some of the variation in the historical sphere eversions based on the Morin-Froissart halfway model.
} 


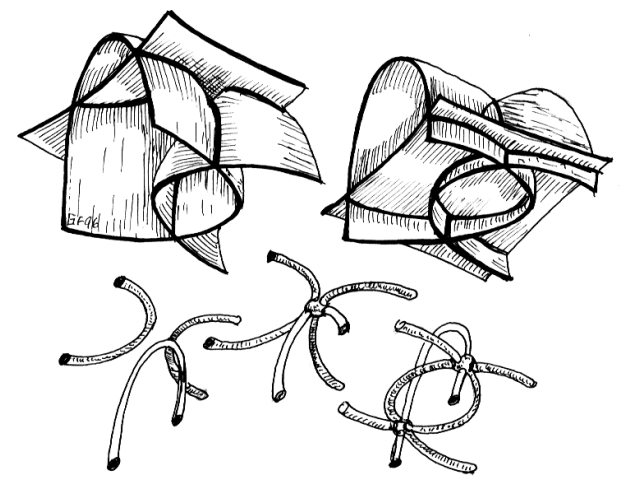

Fig. 3 A model for the birth of triple points in the double locus of an immersed surface. As the water table drops on two partially submerged and intersecting convex surface patches, twin triple points are born simultaneously.
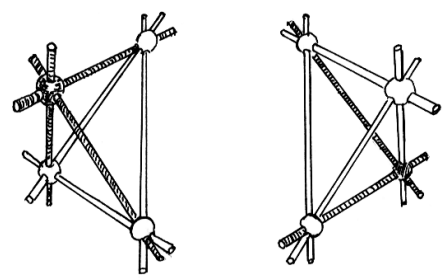

Fig. 4 The double locus on either side of the quadruple point is formed by the planes of a tetrahedron passing simultaneously through the barycenter.
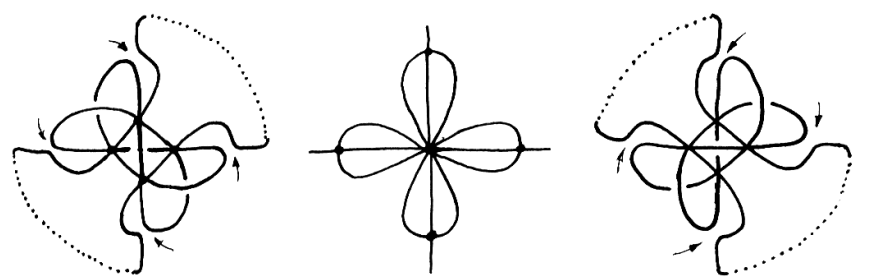

Fig. 5 The double locus of the halfway model drawn schematically as it might be seen from the pole point. Note the quarter-turn symmetry before (left) and after (right) the quadruple point (center).

From each vertex of the tetrahedron emanate 3 double lines that join pairwise into 6 loops. Two triplets of these pass through a pitchfork bifurcation, and the two continuing arches go through the externally visible isthmus at the pole point.

In Fig. 5 we point to the pitchfork bifurcations just before the quadruple point, and just after. All this happens close to $t_{0}=0$. At a more remote time the four triple points die in pairs leaving two disjoint and unlinked unknots, which disappear at the cashew and gastrula $D_{2}$ events. These 
curves do, however, twist about a great deal in space; this is best appreciated in an animation. The death of triple point pairs can be imagined from this schematic as follows. The NW edge of the tetrahedron in Fig. 5(left) is the shortest of three arcs connecting two triple points. Slide it further to the NW, and do the same to the SW portion. This leaves two embedded, unknotted, and unlinked closed loops.

The reader is encouraged to compare Petit's instructive illustrations for a similar eversion in [28]. There, the four $D_{1}$ catastrophes corresponding to our pitchfork bifurcations on the halfway model occur more remotely in time. Two-fold rotational symmetry requires that $t_{0}=t_{1}$ and $t_{2}=t_{3}$; but $t_{0}<t_{2}$, as Petit has it, is not unreasonable.

Using the Apéry-Morin notations [1], the minimax eversion has signature

$$
D_{0} D_{0}\left(T^{+} T^{+}\right)\left(\begin{array}{ccc}
D_{1} & D_{1} & D_{1} \\
D_{1} & Q & D_{1}
\end{array}\right)\left(T^{-} T^{-}\right) D_{2} D_{2} .
$$

The grouping indicates simultaneous occurrence of more than one catastrophe. Time reversals of a $D_{0}$ is denoted by a $D_{2}$ according to the conventions explained in the appendix to [1].

The motion of the double locus, $\mathcal{D}_{t}$ in $\mathbb{R}^{3}$, can be considered the slices of a surface $\hat{\mathcal{D}}$ in $\mathbb{R}^{4}$ given by a Morse function $t: \mathbb{R}^{4} \rightarrow \mathbb{R}$. That way, the five $D_{1}$ correspond to saddles, the two $D_{0}$ to minima, and the two $D_{2}$ to maxima of $t$, for a surface of Euler characteristic -1 . Thus the extruded double locus of the minimax eversion is a Dyck's surface, a torus-with-a-crosscap [9, p. 101]. This is not the 'simplest' possible such double-locus extrusion, according to Apéry and Morin. They construct [1] an eversion whose extruded double locus $\hat{\mathcal{D}}$ is a projective-plane. Their eversion requires additional $D_{0}$ and $D_{2}$ events, however.

\section{Virtual Environments and Supercomputing'95}

We next describe, in the briefest terms, the actual experiment in distributed, parallel computing and its real-time, interactive presentation [13] in the virtual reality media at the joint IEEE-ACM supercomputing conference. A detailed and fully illustrated article on the geometrical graphics we used in our project is in preparation.

The evolver was installed in several 16-processor SGI Power Challenges at the National Center for Supercomputing Applications (NCSA) in Urbana, Illinois and at the Cornell Theory Center (CTC) in Ithaca, New York. These were connected to the convention site in San Diego via any one of several experimental, broad-band, but highly labile network connections. Fig. 6 . We had to be prepared to use whatever was available, at whatever temporal latency. Accordingly, the remotely started evolver used 8 processors (on the Power Challenge of our choice) to compute the successive stages (or topes) of the homotopy, and piped them to the translator process. This shipped the stream to San Diego using the Data Transfer Mechanism (DTM) developed 


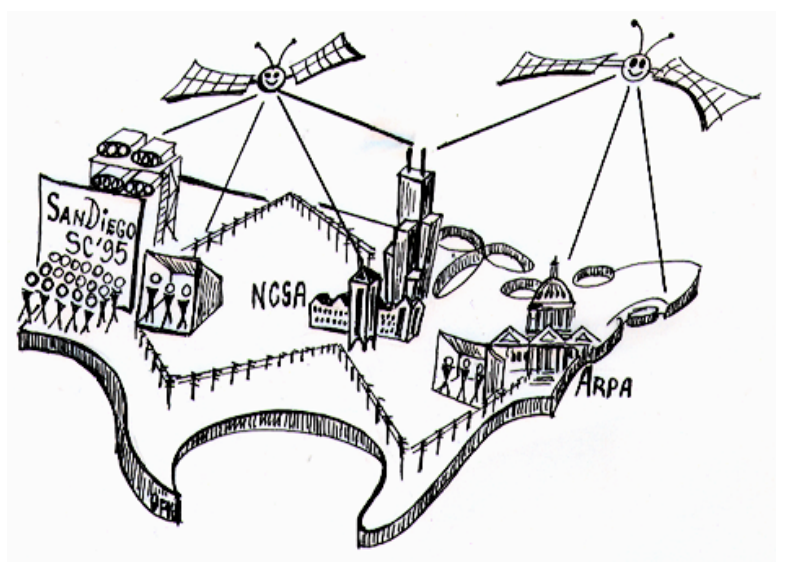

Fig. 6 Cartoon of the I-Way connecting computing and display sites at $\mathrm{Su}-$ percomputing'95. This experimental network, for the real-time interaction of supercomputers and virtual reality displays, was provided by commercial telephone companies.

at the NCSA, on the available line. We found that the ATM line, a vBNS connection designed to reach $155 \mathrm{Mbits} / \mathrm{sec}$, in fact supplied data at only four times the speed of a regular internet connection. ${ }^{17}$ Thus our latencyproof design paid off.

This data flow was sent to the real-time, interactive CAVE application (RTICA) illiVert. ${ }^{18}$ The receiver process of illiVert moved each incoming tope, asynchronously, into the shared memory of the SGI Onyx running the CAVE. ${ }^{19}$ The interactive process of illiVert animated however many topes were currently in memory. Using the temporal symmetry of the eversion on either side of the halfway model, we could investigate the most interesting portion of the eversion right from the start.

Under optimal conditions, it took 7 minutes to complete the calculation and shipment of all 126 topes for the 251-stage eversion ${ }^{20}$ There were actually two CAVEs prepared to communicate with each other transcontinentally, as in Fig. 6. But the CAVE at ARPA headquarters in Washington, DC was not available for our demonstrations.

17 This did not surprise us, because the ATM networks were designed to provide high speed and high bandwidth, intended for live TV transmission.

${ }^{18}$ In addition to the CAVE, there were two display variants using the same software library. The large-screen I-Wall required eight RGB projectors and as many Reality Engines in SGI Onyxes, and the single-projector ImmersaDesk only one of each.

19 The shared memory architecture is appropriate, because each wall of the CAVE is illuminated by separate processes running in parallel on the Onyx.

20 When we first started the experiments six months earlier, it had taken overnight. 


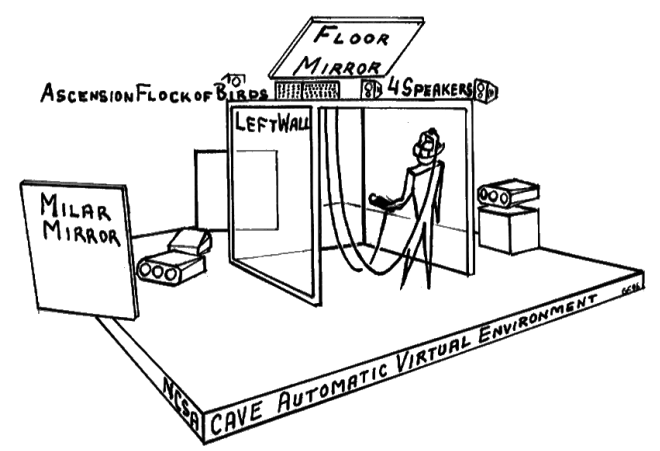

Fig. 7 The arrangement of the CAVE at the National Center for Supercomputing Applications. One projector and two speakers are omitted for a better view.

The CAVE [8] automatic virtual environment, Fig. 7, uses $96 \mathrm{~Hz}$ shutter glasses and a $270^{\circ}$ seamless stereo panorama to evoke a truly immersive 3 -D illusion. The six positional and directional parameters of the viewer's head, and those of the control device (called a wand) are monitored by a Ascension Flock of Birds magnetic tracker. The geometrically accurate (to a few $\mathrm{cm}$ ) perspective on each of three walls and the floor is produced at animation speeds varying from a threshold of 10 frames/sec, to a maximum $48 \mathrm{fps}$. Since each wall needs its own graphics pipeline, ${ }^{21}$ two SGI Onyx computers, connected by Scramnet and HIPPI boards, drive the CAVE. A quadraphonic sound system provides stereo location and spatially accurate attenuation and reverberation using the Vanilla Sound Server (VSS). This is a real-time, software based sound-synthesizer developed at the NCSA [4]. It generated a sound field whose parameters were mapped to the geometrical parameters of illivert.

\section{Acknowledgments}

Francis wishes to thank student collaborators on the illiVert project: coauthors Hartman and Chappell who developed and maintained the code; Will Scullin and Alan Verlo for help with the DTM networking code; Ulrike Axen, Kelly Fitz, Camille Goudeseune and Brian Holloway in Robin Bargar's Sound Development Group at the NCSA for help with the VSS code; Steve Pape and Gary Lindahl at EVL ${ }^{22}$ for help with HIPPI and Scramnet; Alex Bourd and Paul McCreary, who took time from their own projects to help test and improve illiVert.

Sullivan, Kusner and Brakke wish to thank GANG (at the University of Massachusetts), and Sullivan also the Geometry Center (at the University of Minnesota), for support during this project.

\footnotetext{
21 A Silicon Graphics Reality Engine.

22 The Electronic Visualization Laboratory of the University of Illinois at Chicago is the birthplace of the CAVE.
} 


\section{References}

1. F. APÉRY, An algebraic halfway model for the eversion of the sphere, Tohoku Math. J. 44 (1992), 103-150, with an appendix by B. Morin.

2. _ Le retournement du cuboctaèdre, preprint, Institute de Recherche Mathématique Avancée, U. Louis Pasteur, Strasbourg, 1994.

3. T. BANChOFF AND N. L. MAX, Every sphere eversion has a quadruple point, Contributions to Analysis and Geometry (Baltimore, MD, 1980) (D. N. Clark, G. Pecelli, And R. SACksteder, eds.), Johns Hopkins Univ. Press, Baltimore, 1981, pp. 191-209.

4. R. Bargar, I. Choi, S. Das, and C. Goudeseune, Model-based interactive sound for an immersive virtual environment, Proceedings of the International Computer Music Conference (Aarhus, Denmark), International Computer Music Association, 1994, pp. 471-474.

5. K. A. Brakke, The surface evolver, Experimental Mathematics 1:2 (1992), 141-165.

6. K. A. Brakke AND J. M. Sullivan, Using symmetry features of the surface evolver to study foams, Mathematics and Visualization (K. PolthiER AND H.-C. Hege, eds.), Springer Verlag, Berlin, 1996, pp. ???-???

7. R. BRyant, A duality theorem for Willmore surfaces, J. Differential Geometry 20 (1984), 23-53.

8. C. Cruz-Neira, D. J. Sandin, T. A. DeFanti, R. V. Kenyon, and J. C. HART, The CAVE: Audio-visual experience automatic virtual environment, Communications ACM 35:6 (1992), 65-72.

9. G. Francis, A topological picturebook, Springer-Verlag, New York, 1987.

10. - The hypergraphics honors seminar at Illinois, Scientific Visualization in Mathematics and Science Teaching (D. Thomas, ed.), Assoc. Adv. Comp. in Educ., Charlottesville, VA, 1995.

11. G. Francis, F. Apéry, C. Hartman, and G. Chappell, Equivariant sphere eversions, narrated videotape (9 min) produced at the NCSA, U. Illinois, 1992.

12. G. Francis And B. Morin, Arnold Shapiro's eversion of the sphere, Math. Intelligencer 2 (1979), 200-203.

13. G. Francis, J. M. Sullivan, K. Brakke, R. Kusner, D. Roseman, A. Bourd, C. Hartman, G. Chappell, and J. Rubenstein, Laterna matheMAGICA, Virtual Environments and Distributed Computing at SC'95: GII Testbed and HPC Challenge Applications on the I-WAY (H. KorAB AND M. D. Brown, eds.), ACM/IEEE Supercomputing'95, 1995.

14. A. Hanson, T. Munzner, And G. Francis, Interactive methods for visualizable geometry, IEEE Computer 27:4 (1994), 73-83.

15. A. E. Hatcher, A proof of the Smale conjecture $\operatorname{Diff}\left(S^{3}\right) \simeq O(4)$, Annals of Math. 117 (1983), 553-607.

16. J. Hoffman And R. Kusner, Elastic surfaces and conformal geometry, video, produced at GANG, U. Massachusetts, Amherst, MA, 1992.

17. L. Hsu, R. Kusner, And J. M. Sullivan, Minimizing the squared mean curvature integral for surfaces in space forms, Experimental Mathematics 1:3 
(1992), 191-207.

18. J. F. HuGHES, Another proof that every eversion of the sphere has a quadruple point, Amer. J. Math. 107:2 (1985), 501-505.

19. Polynomial models of smooth surfaces, presentation at the conference on Computer Graphics in Pure Mathematics (Dennis Roseman, organizer), U. Iowa and Inst. for Mathematics and its Applications, 1990.

20. R. Kusner, Conformal geometry and complete minimal surfaces, Bull. Amer. Math. Soc. 17 (1987), 291-295.

21. R. Kusner AND N. Schmitt, The spinor representation of minimal surfaces, GANG preprint, 1994.

22. S. Levy, Making waves: A guide to the ideas behind Outside In, A K Peters, Wellesley, MA, 1995.

23. S. Levy, D. Maxwell, And T. Munzner, Outside In, A K Peters, Wellesley, MA, 1994, narrated videotape $(21 \mathrm{~min})$ produced by the Geometry Center, University of Minnesota.

24. P. LI AND S. T. YAU, A new conformal invariant and its applications to the Willmore conjecture and the first eigenvalue of compact surfaces, Invent. Math. 69 (1982), 269-291.

25. N. L. MAx, Turning a sphere inside out, International Film Bureau, Chicago, 1977, narrated videotape (21 $\mathrm{min})$.

26. Computer animation in mathematics, science, and art, Computers in Mathematics (D. V. Chudnovsky And R. D. Jenks, eds.), Lecture Notes in Pure and Applied Mathematics, vol. 125, Marcel Dekker, Inc., New York, 1990, includes as an appendix the illustrated guide to [25], pp. 321-345.

27. B. Morin, Équations du retournement de la sphére, Comptes Rendus Acad. Sci. Paris 287 (1978), 879-882.

28. B. Morin And J.-P. Petit, Le retournement de la sphère, Les Progrès des Mathématiques, Pour la Science/Belin, Paris, 1980, pp. 32-45.

29. A. Phillips, Turning a sphere inside out, Sci. Amer. 214 (1966), 112-120.

30. S. Smale, A classification of immersions of the two-sphere, Trans. Amer. Math. Soc. 90 (1959), 281-290.

31. T. J. Willmore, Note on embedded surfaces, An. Stiint. Univ "Al. I. Cuza" Iasi Sect. I, a Mat. 11 (1965), 493-496. 

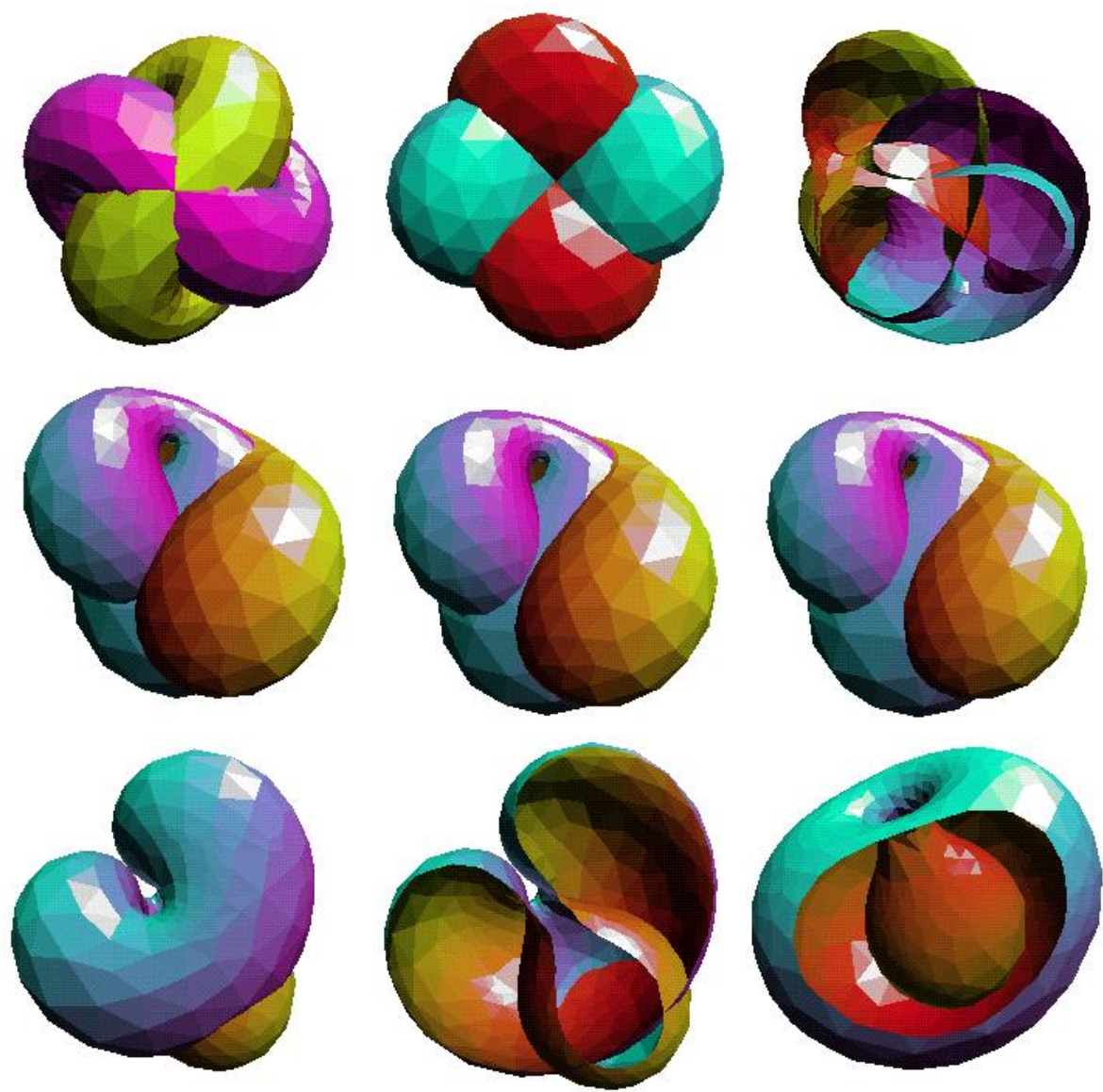

Fig. 8 This figure shows stages of the minimax eversion. The top row has views of the halfway model, showing the pole point (left) and the quadruple point (middle); the internal complexity of the halfway model is seen in the cut-away image (right). The middle row shows the death of a pair of triple points. In the bottom row, we see the death of a double curve (left and middle), and an embedded sphere in the shape of a gastrula near the end of the eversion (right). (G. Francis, J. M. Sullivan, et al, p. 9.) 

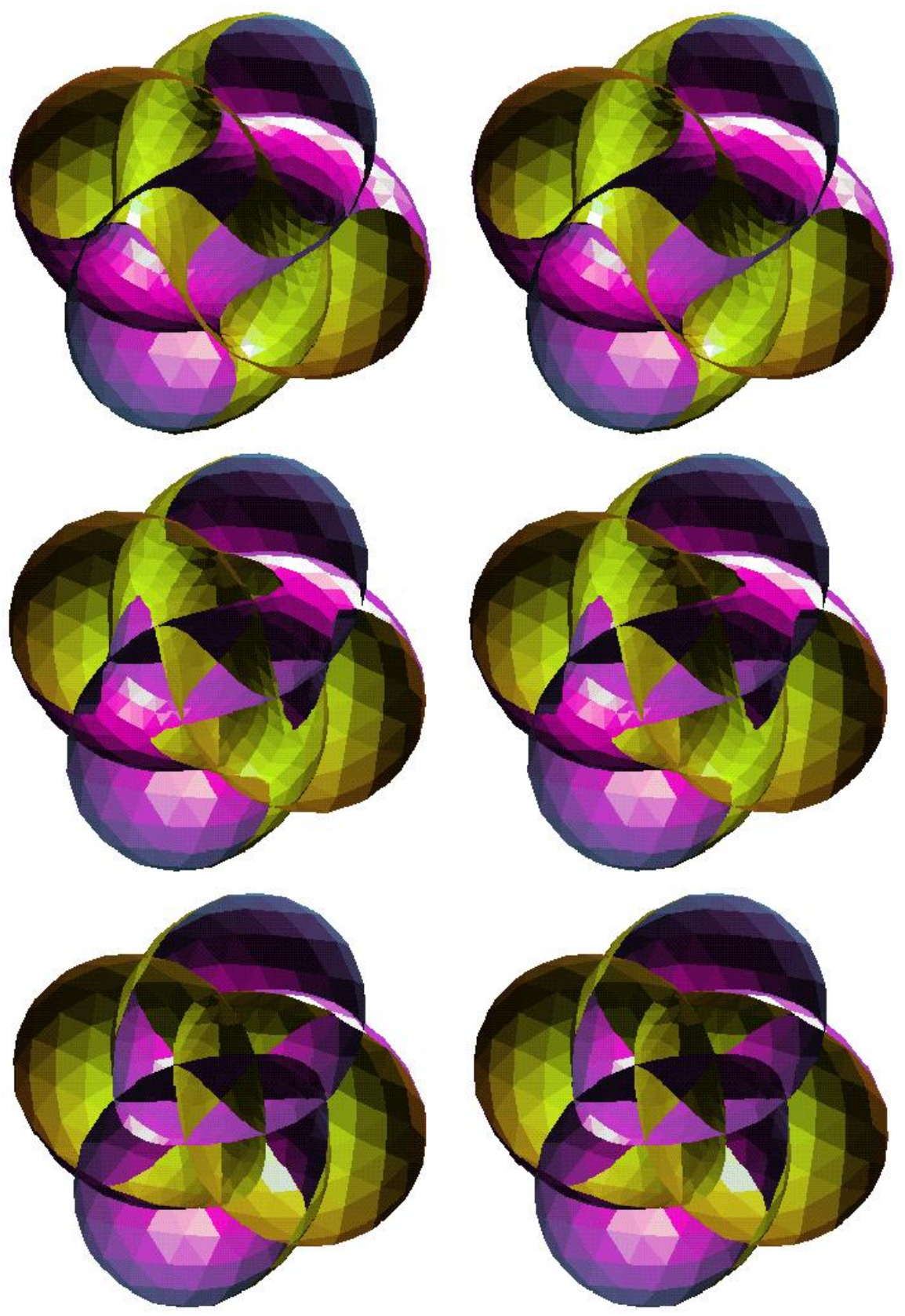

Fig. 9 This figure shows three stereo pairs (printed for cross-eyed viewing), giving cut-away views of the halfway model for the minimax sphere eversion, looking along the symmetry axis towards the quadruple point. From top to bottom, we slice further and further from the eye: at the top we have removed just two cap-like disks through the pole point, while at the bottom we leave four bowl-like disks crossing at the quadruple point. (G. Francis, J. M. Sullivan, et al, p. 10.) 